\title{
Relativistic mass of gravitational field
}

\author{
Yin Zhu \\ Agriculture and Rural Department of Hubei Province, Wuhan, China \\ Email:waterzhu@163.com
}

(November 19, 2021)

\begin{abstract}
It is discovered that the gravitational field on the surface of a neutron star is with a relativistic mass density of $2.65 \times 10^{16} \sim 5.87 \times 10^{18} \mathrm{kgm}^{-3}$ which can be larger than the mass density of the neutron stars $\left(\sim 10^{17} \mathrm{kgm}^{-3}\right)$. And, the total relativistic mass of the gravitational field of the Sun is $\sim 10^{7}$ times the mass of the Sun. For different stars, the relativistic mass of the gravitational field is larger as the mass density of the star is larger. In the Milky Way, the total relativistic mass of the gravitational fields is much larger than the total mass of the stars, planets and gas. And, the relativistic mass density of the observed strongest magnetic field is $2.17 \times 10^{8} \mathrm{kgm}^{-3}$. This discovery should be a new frame to understand the fundamental problem of physics.
\end{abstract}

Key Words: Relativistic mass of gravitational field; Total mass of a galaxy; Constitution of the mass of a star with field 
For a body $\mathrm{m}$ freely falling in a gravitational field of $\mathrm{M}$, there is $\mathrm{mgh}=\frac{1}{2} m v^{2}$, and for a celestial body $\mathrm{m}$ orbiting around $\mathrm{M}$, there is $\frac{1}{2} m v^{2}=G \frac{M m}{R}$. The energy of a gravitational field can make a body moved with an energy of $\mathrm{E}=\frac{1}{2} m v^{2}$ and is the reason to make the neutrons degenerated to form the neuron star. Currently, it is thought that the concept of gravitational potential energy of a star is correct and useful.[1] The energy density, $\mathrm{U}=\frac{g^{2}}{8 \pi G}$, of a gravitational field on the surface of a star is determined with the gravitational acceleration $g$ and Newtonian universe gravitational constant G.[1,2] From $\mathrm{E}=\mathrm{m} c^{2}$, it is well known that a gravitational field is with a relativistic mass density $d_{g}=\frac{1}{c^{2}} \frac{g^{2}}{8 \pi G}$. Here, it is discovered that the relativistic mass density of the gravitational field on the surface of a neutron star is $2.65 \times 10^{16} \sim 5.87 \times 10^{18} \mathrm{kgm}^{-3}$ which can be larger than the mass density of the neutron stars $\left(\sim 10^{17} \mathrm{kgm}^{-3}\right)$.[3,4] And, the total relativistic mass of the gravitational field of the Sun or a neutron star is $\sim 10^{7}$ times the mass of the Sun or is $\sim 10^{21}$ times that of the neutron star. For different stars, the relativistic mass of the gravitational field is larger as the mass density of the star is larger. And, the relativistic mass density of the observed strongest magnetic field is $d_{m}=\frac{1}{c^{2}} \frac{B^{2}}{2 \mu_{0}} \approx 2.17 \times 10^{8} \mathrm{kgm}^{-3}$. This discovery should be a new frame to understand the fundamental problem of physics.

\section{The relativistic mass density and total relativistic mass of a gravitational field}

On the surface of the Earth, $g_{e}=10 \mathrm{~ms}^{-2}$, correspondently, $U_{g e}=\frac{g^{2}}{8 \pi G} \approx 5.97 \times 10^{10} \mathrm{kgm}^{-1} s^{-2}$. In this case, $d_{g e}=\frac{1}{c^{2}} \frac{g^{2}}{8 \pi G} \approx 6.63 \times 10^{-7} \mathrm{kgm}^{-3}$ is very little. Therefore, it is usually neglected in current physics. For example, the mass density of the hydrogen gas is $d_{H}=8.9887 .5 \times 10^{-2} \mathrm{kgm}^{-3}$. Therefore, the gravitational field on the surface of the Earth with the relativistic mass density of $d_{g e}=\frac{1}{c^{2}} \frac{g^{2}}{8 \pi G} \approx 6.63 \times 10^{-7} \mathrm{kgm}^{-3}$ has little effect on the mass density of the hydrogen gas. But, for a super strong gravitational field (Here, we call the gravitational field with $d_{g}=\frac{1}{c^{2}} \frac{g^{2}}{8 \pi G} \geq 1 \times 10^{3} \mathrm{kgm}^{-3}$ the super strong field), the energy density and relativistic mass density are very big. For example, the gravitational acceleration on the surface of a neutron star is $g_{n}=$ $2 \times 10^{11} \sim 3 \times 10^{12} g_{e} .[5,6] \quad$ Correspondently, there are $\mathrm{U}_{g n}=\frac{g^{2}}{8 \pi G} \approx 2.39 \times 10^{33} \sim 5.37 \times$ $10^{35} \mathrm{kgm}^{-1} \mathrm{~s}^{-2}$ and $d_{g n}=\frac{1}{c^{2}} \frac{g^{2}}{8 \pi G} \approx 2.65 \times 10^{16} \sim 5.90 \times 10^{18} \mathrm{kgm}^{-3}$. We know, the density of water is $10^{3} \mathrm{kgm}^{-3}$; the largest mass density in the Earth is $2.259 \times 10^{4} \mathrm{~kg} / \mathrm{m}^{3}$ (the osmium). The relativistic mass density of the gravitational field on the surface of a neutron star is about $10^{13} \sim 10^{15}$ times the density of the water and $10^{12} \sim 10^{14}$ times the largest mass density in the Earth. The author is extremely astonished and fascinated by this discovery. It shows that the relativistic mass of the gravitational field is very important to well understand the total mass in a galaxy. 
It is very significant and interesting that the relativistic mass density of the gravitational field of a neutron star can be larger than the mass density of the neutron stars.

For a star with the relativistic mass density $d_{g}=\frac{1}{c^{2}} \frac{g^{2}}{8 \pi G}$ of the gravitational field and the mass density of the star $d_{m}=M /\left(\frac{4}{3} \pi r^{3}\right)$, for $d_{g} \geq d_{m}$, there is

$$
G \frac{M}{r} \geq 6 c^{2}
$$

or

$$
\mathrm{r} \leq \frac{1}{6} \frac{G M}{c^{2}}
$$

$r_{s}=\frac{2 G M}{c^{2}}$ is the Schwarzschild radius. It indicates that, as the radius of a star is less than $\frac{1}{12}$ times the Schwarzschild radius, the relativistic mass density of the gravitational field on the surface of the star is larger than the mass density of the star.

From the energy density, the total relativistic mass of a gravitational field can be known.

As shown in Figure 1A, the energy density $U=\frac{g^{2}}{8 \pi G}$ is determined with the radius $r$ of the star. In the volume of $\mathrm{dV}=\frac{4}{3} \pi\left[(r+\mathrm{d} r)^{3}-r^{3}\right]$, because of $g=\mathrm{G} \frac{M}{r^{2}}$, the energy density $\mathrm{U}=\frac{g^{2}}{8 \pi G}$ is not uniform. But, as the $d r$ is very little, approximately, the average energy density can be determined with $g=g(\mathrm{r}) \approx g(r+d r)$. The energy density in $\mathrm{dV}$ can be treated as uniform. Therefore, in $\mathrm{dV}$, the energy density can be taken approximately as $\mathrm{U}=\frac{g^{2}}{8 \pi G}$. In this sense, the energy density in the whole field can be described with a line as shown in Figure 2A. The energy in $\mathrm{dV}$ can be simply written as $\mathrm{dE}=d \frac{g^{2}}{8 \pi G} d V$. Therefore, the total energy in the whole volume $\mathrm{V}=\frac{4}{3} \pi R^{3}$ is $\mathrm{E}=\iint d \frac{g^{2}}{24 \pi G} d V$. It is clear, the total energy in V also is $\mathrm{E}=\bar{U} V$, where $\bar{U}$ is the average energy density of the field in the whole field V. From $U=\frac{g^{2}}{8 \pi G}$ and $g=\mathrm{G} \frac{M}{r^{2}}$, in Supplementary Information 1, it is obtained that the average energy density is

$$
\bar{U}=\frac{g^{2}}{24 \pi G} \frac{r}{R}
$$

where $\mathrm{r}$ is the radius of the star, $\mathrm{R}$ is the radius of the gravitational field as shown in Figure 1A. Therefore, from $\mathrm{E}=$ $\bar{U} V$ and $\mathrm{V}=\frac{4}{3} \pi R^{3}$, the total energy of the gravitational field of a star is $\mathrm{E}=\frac{g^{2}}{18 G} r R^{2}$. 
A

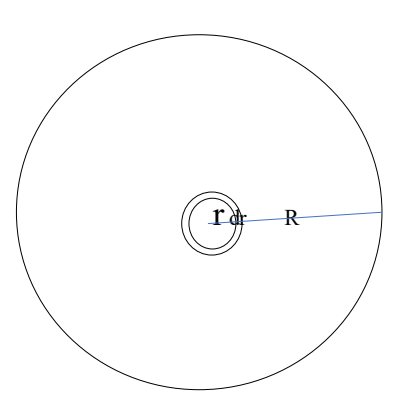

B

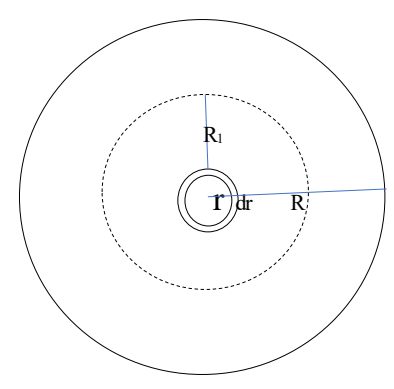

Figure 1. The energy density of a gravitational field. A. $r$ is the radius of the star. $R$ is the radius of the gravitational field. $d r$ is a height above the surface of the star and $\mathrm{dr} \rightarrow 0$. Therefore, there is the $g=g(r) \approx g(r+d r)$. The energy density in the volume of $\mathrm{dV}=\frac{4}{3} \pi\left[(r+d r)^{3}-\right.$ $r^{3}$ ] can be approximately written as $\mathrm{U}(\mathrm{dr})=\frac{g^{2}}{8 \pi G}$. B. The total energy in the volume $\mathrm{V}=\frac{4}{3} \pi R^{3}$ is larger than that in $V_{1}=\frac{4}{3} \pi R_{1}^{3}$.

$\mathrm{U}=\frac{g^{2}}{8 \pi G}$ is related with $\frac{1}{R^{4}}$. Therefore, it is reduced sharply as shown in Figure 2A. For a certain $g$, the average energy density $\bar{U}=\frac{g^{2}}{24 \pi G} \frac{r}{R}$ is a proportional to $\frac{1}{R}$ as shown in Figure 2B. From Figure 1, it can be clearly known that, as $\mathrm{R}$ is increased, the energy is correspondently increased. The total energy $\mathrm{E}=\frac{g^{2}}{18 G} r R^{2}$ is a proportional to $R^{2}$ as shown in Figure 2C. The increased rate of the total energy is exponential. 
A

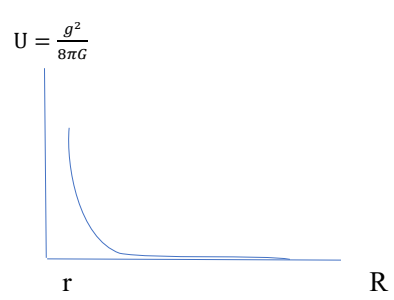

B

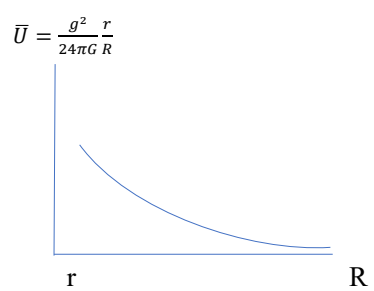

C

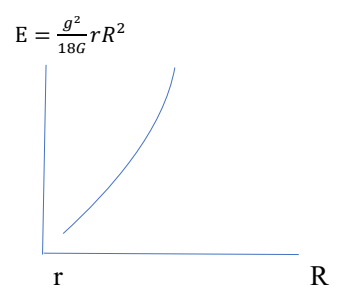

Figure 2. The energy density, average energy density and the total energy of a gravitational field. $\mathrm{r}$ is the radius of the star. $\mathrm{R}$ is the radius of the field. A. The energy density. In the case of $g=g(r) \approx$ $g(r+d r)$, in the volume $\mathrm{dV}=\frac{4}{3} \pi\left[(r+d r)^{3}-r^{3}\right]$, the gravitational field can be treated as a uniform field. The energy density can be expressed as $U=\frac{g^{2}}{8 \pi G}$. B. The average energy density. As $\Delta \mathrm{r}$ is large, $g(r)>g(r+\Delta r)$, in the volume of $\Delta \mathrm{V}=\frac{4}{3} \pi\left[(r+\Delta r)^{3}-r^{3}\right]$, the gravitational field cannot be treated as a uniform one, the average energy density $\bar{U}=\frac{g^{2}}{24 \pi G} \frac{r}{R}$ can be obtained from $\mathrm{U}=$ $\frac{g^{2}}{8 \pi G}$. C. The total energy. The total energy is the sum of the energy in the whole gravitational field. The volume of the gravitational field is determined with $\mathrm{V}=\frac{4}{3} \pi R^{3}$. Therefore, the total energy is a proportional to $R^{3}$. Because the average energy density $\bar{U}=\frac{g^{2}}{24 \pi G} \frac{r}{R}$ is a proportional to $1 / \mathrm{R}$, thus, the total energy is at last a proportional to $R^{2}$

Now, it is believed that, the radius of a gravitational field is infinite. Therefore, in current theory, the relativistic mass of any gravitational field should be infinite large. However, that is not true.

It is noted that, the energy density, $\mathrm{U}=\frac{g^{2}}{8 \pi G}$, of a gravitational field is determined with the gravitational acceleration $g$ of the field. Thus, as the action of a gravitational field on another ones is not $g$, the boundary of the gravitational field can be defined. From Newtonian orbital perturbation theory, we know, out of the Hill radius, the gravitational acceleration of a celestial body on others is not $\mathrm{g}=\mathrm{G} \frac{M}{R^{2}} \cdot[7,8,9]$ Therefore, the total energy of a 
gravitational field could be written as

$$
\mathrm{E}=\frac{g^{2}}{18 G} r R_{H}^{2}
$$

Where $R_{H}$ is the Hill radius of the star or planet.

It is noted that, Equation (4) only is valid to describe a special case of the energy of a gravitational field, i.e., the total energy of the gravitational field inside the Hill sphere of the field. Therefore, the energy of the field out of the Hill sphere need be further studied elsewhere.

From Equation (4) we know, the total relativistic mass $m_{\text {field }}$ of a gravitational field is:

$$
m_{\text {field }}=\frac{1}{c^{2}} \frac{g^{2}}{18 G} r R_{H}^{2}
$$

From Equation (5), the relativistic mass of the field of the Sun, Earth and Neptune it is calculated as shown in the Table 1 .

Table 1: The relativistic mass of gravitational fields in the solar system

\begin{tabular}{|l|l|l|l|}
\hline & Sun & Earth & Neptune \\
\hline $\mathrm{m}_{\text {field }}(\mathrm{kg})$ & $4.30 \times 10^{37}$ & $1.28 \times 10^{19}$ & $8.03 \times 10^{24}$ \\
\hline $\mathrm{M}(\mathrm{kg})$ & $1.989 \times 10^{30}$ & $5.97 \times 10^{24}$ & $1.02 \times 10^{26}$ \\
\hline $\mathrm{m}_{\text {field }} / \mathrm{M}$ & $2.16 \times 10^{7}$ & $2.14 \times 10^{-6}$ & $7.87 \times 10^{-2}$ \\
\hline $\mathrm{R}_{\mathrm{H}}$ & 1 light year & $1.5 \times 10^{9} \mathrm{~m}$ & $5.4 \times 10^{11} \mathrm{~m}$ \\
\hline$g\left(\mathrm{~m} / \mathrm{s}^{2}\right)$ & 273.20 & 9.80 & 11.15 \\
\hline
\end{tabular}

It is noted that, the Hill radius of the Sun has not been known. Here, for the Sun, the $R_{H}$ is taken as the distance between the Sun and the Oort Cloud which now is thought to be the boundary of the Sun's gravitational influence.[10] 
But, it is clear, the distance for the action of the Sun on other body with $g=G \frac{M}{R^{2}}$ is larger than that on the Oort cloud. So, the relativistic mass of the field of the Sun is larger than $4.3 \times 10^{37} \mathrm{~kg}$.

The Table 1 shows: 1) the relativistic mass of the gravitational field of a celestial body could be larger or less than the mass of the body. 2) the relativistic mass of the gravitational field of the solar system is $10^{7}$ times the total mass of the Sun and the planets in the system for that the mass of the Sun is larger than $90 \%$ of total mass of the bodies and gas in the solar system.

From Equation (5) we know, for the stars with the same mass, the relativistic mass of the gravitational fields is related with the mass density of the stars. From $\mathrm{M}=\rho \mathrm{v}$, where $\rho$ and $\mathrm{v}=\frac{4}{3} \pi r^{3}$ are the density and volume of the star, and from $m_{\text {field }}=\frac{1}{c^{2}} \frac{g^{2}}{18 G} r R_{H}^{2}$ and $g=\mathrm{G} \frac{M}{r^{2}}$, the relationship between the relativistic mass of the gravitational field and the density $\rho$ of the star can be expressed as

$$
m_{\text {field }}=\frac{1}{c^{2}} \frac{2 \pi^{2} \mathrm{G}}{81} r^{3} R^{2} \rho^{2}
$$

For the stars with different mass density and with the mass of 1 Sun and the gravitational boundary of $\mathrm{R}=1$ light year, the relativistic masses of the gravitational fields of them are shown in the Table 2:

Table 2. The density of the stars and the relativistic mass of the fields

\begin{tabular}{|l|l|l|l|l|}
\hline & Red giant star[11] & Sun & White dwarf star[12] & Neutron star[3,4] \\
\hline$\rho\left(10^{3} \mathrm{~kg} / \mathrm{m}^{3}\right)$ & $10^{-2}$ & 1.4 & $10^{6}$ & $4.75 \times 10^{14}$ \\
\hline $\mathrm{r}(\mathrm{m})$ & $3.62 \times 10^{10}$ & $6.96 \times 10^{8}$ & $7.81 \times 10^{7}$ & $1.00 \times 10^{4}$ \\
\hline$g\left(\mathrm{~m} / \mathrm{s}^{2}\right)$ & $1.01 \times 10^{-1}$ & $2.73 \times 10^{2}$ & $2.19 \times 10^{4}$ & $1.33 \times 10^{12}$ \\
\hline $\mathrm{m}_{\text {field }}(\mathrm{kg})$ & $3.06 \times 10^{32}$ & $4.30 \times 10^{37}$ & $3.10 \times 10^{40}$ & $1.47 \times 10^{52}$ \\
\hline $\mathrm{m}_{\text {field }} / \mathrm{M}$ & $1.54 \times 10^{2}$ & $2.16 \times 10^{7}$ & $1.55 \times 10^{10}$ & $7.39 \times 10^{21}$ \\
\hline
\end{tabular}

From Table 2, we have Figure 3 


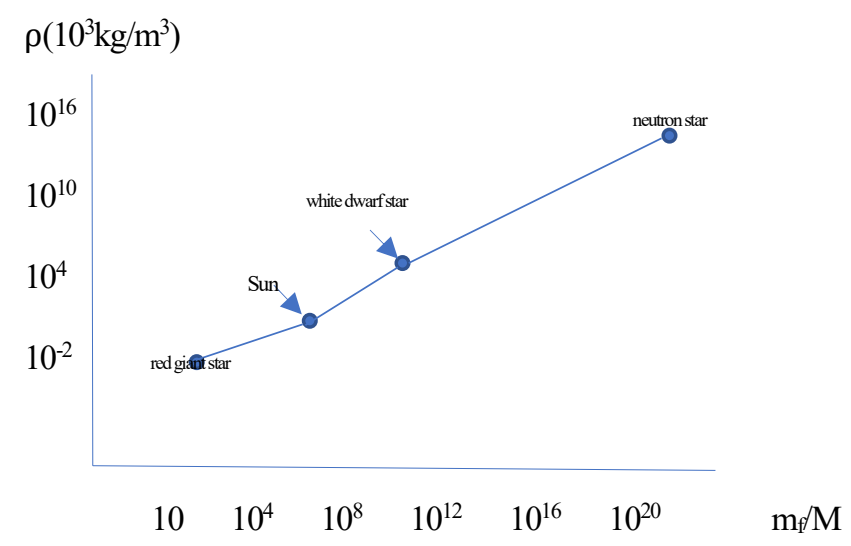

Figure 3. The density of the stars and the relativistic mas of the field of them. The red giant star, Sun, white dwarf star and neutron star are with the mass of 1 Sun and the radius of the gravitational field is $\mathrm{R}=1$ light year. The density of the neutron star is almost $10^{16}$ times that of the red giant star while the relativistic mass of the field of neutron star is almost $10^{20}$ times that of the red giant star.

Table 2 and Figure 3 is helpful to estimate the total relativistic mass of the gravitational fields in a galaxy.

The Sgr A* of the central of the Milky Way is with the mass of $4 \times 10^{6}$ times that of the Sun and the radius of $2.2 \times 10^{10} \mathrm{~m}$.[13] The gravitational acceleration on the surface of the Sgr A* is $g=1.1 \times 10^{6} \mathrm{~m} / \mathrm{s}^{2}$. The gravitational boundary of the Sgr $\mathrm{A}^{*}$ is almost $10^{5}$ light year. From Equation (2), it can be calculated that the relativistic mass of the field of Sgr $A *$ is $m_{\text {Sgrafield }}=7.01 \times 10^{55} \mathrm{~kg}$ and $m_{\text {SgrAfield }} / M_{\text {SgrA }} \approx 8.81 \times 10^{18}$. There are almost $5.8 \times 10^{11}$ stars in the Milky Way. The Table 2 shows, the relativistic mass of the neutron star is almost $10^{21}$ times the Sun. But, up to now only $\sim 2 \times 10^{3}$ neutron star have been observed.[14] Therefore, the relativistic mass of the field of the Sgr A* is larger than the sum of both the masses of the observed red giant stars, white dwarf stars and neutron stars and their fields in the Milky Way.

Table 2 and Figure 3 and $m_{\text {Sgrafield }} / M_{\text {SgrA }} \approx 8.81 \times 10^{18}$ should indicate that, in a galaxy, the total mass of the celestial bodies and gas is much less than the total relativistic mass of the gravitational fields in the same galaxy. 


\section{Discussions and conclusions}

The energy density of the gravitational field on the surface of a star was presented early in 1950.[2] The energy density of the gravitational field of a star is not uniform. Instead, it is varied with $\frac{1}{R^{4}}$ as shown in Figure $2 \mathrm{~A}$. Therefore, the average energy density of the field is needed to obtain the sum of the total energy in the whole volume of the field. But, the average energy density has not been known till know. In this work, the average energy density of the gravitational field is first studied.

From the average energy density of a gravitational field, in this work, the total energy of a whole gravitational field is first calculated. It is noted that the formula $\mathrm{E}=\frac{1}{2} \frac{G M^{2}}{R}[1,2,15]$ is only the energy in the field of $\mathrm{dr} \rightarrow 0$ above the surface of the star. In the case, the average energy density in the sphere can be expressed as $U=\frac{g^{2}}{8 \pi G}$, where $g=g(r)$ as discussed in the Figure 1. And, it is known that the energy $\mathrm{E}=\frac{3}{5} \frac{G M^{2}}{R}[16,17]$ is the gravitational self-attracted energy which makes the neutrons degenerated to form the neutron star. Therefore, $\mathrm{E}=$ $\frac{1}{2} \frac{G M^{2}}{R}$ or $\mathrm{E}=\frac{3}{5} \frac{G M^{2}}{R}$ is not suitable to calculate the total energy of one whole gravitational field. If it should be valid for the total energy of the whole gravitational field, the total energy in a whole field should be less than the part energy in the part field. For example, in the Figure 1B, if $\mathrm{E}=\frac{1}{2} \frac{G M^{2}}{R}$ should be valid, there is $E_{R}=\frac{1}{2} \frac{G M^{2}}{R}<E_{R 1}=\frac{1}{2} \frac{G M^{2}}{R_{1}}$. That is clearly not true.

The observed strongest magnetic field is with the magnetic flux density of $\mathrm{B} \approx 7 \times 10^{9} \mathrm{~T}$.[18,19,20] From the energy density of the magnetic field $U=\frac{B^{2}}{2 \mu_{0}}$, it is known that the relativistic mass density of this magnetic field is $d_{m}=\frac{1}{c^{2}} \frac{B^{2}}{2 \mu_{0}} \approx 2.17 \times 10^{8} \mathrm{kgm}^{-3}$. Usually, the radius of the neutron star is $\sim 10^{4} \mathrm{~m}$ and the "radius" of the magnetic field of a star is about 10 times the radius of the star. From $\mathrm{V}=\frac{4}{3} \pi R^{3}$, we know, as the "radius" of the magnetic field is $\mathrm{R}=1 \times 10^{5} \mathrm{~m}$, there is $\mathrm{V}=\frac{4}{3} \pi \times 10^{15} \mathrm{~m}^{3}$. Thus, the relativistic mass of the magnetic field of the neutron star could be $m_{\text {magneticfield }}=9.1 \times 10^{23} \mathrm{~kg}$ which is $\sim \frac{1}{10}$ times the mass of the Earth. However, it is noted that this is a very rough estimation: 1 ) the magnetic field is not uniform. $\mathrm{B} \approx 7 \times 10^{9} \mathrm{~T}$ is not suitable for the whole magnetic field. 2) the volume of the magnetic field has not been accurately known. But, this estimation can imply that the relativistic mass of the magnetic field of a neutron star could be larger than the mass of the star.

Therefore, the two natures: 1) the relativistic mass density of field is very large and 2) the relativistic mass of the field can be larger than the mass of the star, are not unique to the super strong gravitational field, but is common to 
the super strong magnetic field. Because the gravitational field is full of all the space while the magnetic field only exist in the part space, and, the relativistic mass density and the total relativistic mass of the gravitational field of the neutron star is much larger than that of the strongest magnetic field, the gravitational field is more fundamental to the mass of a galaxy.

It is well known by almost every physicist that, according to $\mathrm{E}=\mathrm{m} c^{2}$, a field is with a relativistic mass. But, it is the first time that the relativistic mass of the gravitational and magnetic fields is calculated in this work. The result is extremely astonishing and fascinating: The relativistic mass of the gravitational or magnetic field of a star can be much larger than the mass of the star.

This result shows a new frame to understand the total mass of a galaxy. It indicates that, in a galaxy, the total mass of the bodies and gas is very little. Now, the total mass of the Milky Way is estimated to be $5.8 \times 10^{11}$ times that of the Sun.[21] From Table 2 and Figure 3, it is shown that the relativistic mass of the gravitational field of a neutron star could be $7.39 \times 10^{21}$ times the mass of the Sun. It is much larger than the total mass of the stars and gas in the Milky Way. Although it is astonishing, it is understandable. It is easy to know that the mass density in the Milky Way is only $D_{M Y}=2.5 \times 10^{-17} \mathrm{~kg} / \mathrm{m}^{3}$ as the total mass in the Milky way is taken as $5.8 \times 10^{11}$ times the Sun and the radius of the Milky Way is $5 \times 10^{4}$ light year. While the average relativistic mass density of the gravitational field of the neutron star is $\overline{D_{n}}=\frac{1}{c^{2}} \frac{g^{2}}{24 \pi G} \frac{r}{R}=4.13 \times 10^{4} \mathrm{~kg} / \mathrm{m}^{3}$. This is a very large density. It is larger than the largest mass density in the Earth which is $2.259 \times 10^{4} \mathrm{~kg} / \mathrm{m}^{3}$ of the osmium. But, it was discovered, the relativistic mass density of the gravitational field on the surface of a neutron star is approximately $d_{g n}=\frac{1}{c^{2}} \frac{g^{2}}{8 \pi G} \approx 2.65 \times 10^{16} \sim 5.87 \times 10^{18} \mathrm{kgm}^{-3}$ which can be larger than the mass density of the neutron stars. So, although the average relativistic mass density of the gravitational field of the neutron star is so large that is far beyond expectation, it is possible.

A new frame is needed to understand both that the relativistic mass density of the gravitational field on the surface of a neutron star can be larger than the mass density of the star and that relativistic mass of the gravitational field of a star is much larger than the mass of the star.

In the new frame, the main mass in a galaxy should be the relativistic mass of the gravitational field. As we consider the mass of a galaxy or the whole universe, not only the mass of the bodies and gas is important but also the 
relativistic mass of the field is significant.

The two problems are very interesting and important:

1) For body (including star and planet) and gravitational field, which one is the premise? It is usually thought that a gravitational field is emitted by a body. But, now, it is known that the relativistic mass of the gravitational field of a body is much larger than the mass of the body. Why a body can emit a field with the mass that is much larger than the mass of the body?

2) What is the mechanism that can make the matter with the relativistic mass density larger than the mass density of the neutron star a field?

\section{References}

[1] Lyinden-Bell D. \& Kartz J., Gravitational field energy density for spheres and black holes, Mon. Not. Astro. Soc., 213, (1985)

[2] Miller J. S., On the Energy Density in a Gravitational Field, The American Journal of Physics, 18 (4) (1950)

[3] Lattimer J. M., and Prakash M., Neutron Star Structure and the Equation of State, The Astrophysical Journal 550.1(2000)

[4] Vidaña I., Short introduction to the physics of neutron stars, EPJ Web of Conferences 227, 01018 (2020)

[5] Bejger M. \& Haensel P., Surface gravity of neutron stars and strange stars, Astron. Astrophys. 420, 987 (2004)

[6] Gendy M. A. \& Morsink S. M., Universality of the Acceleration due to Gravity on the Surface of a Rapidly Rotating Neutron Star, The Astrophys. J., 791, 78 (2014)

[7] Newton I., Philosophiae Naturalis Principia Mathematica, (Harvard University, Cambridge, MA, 1972)

[8] Gutzwiller M. C., Moon-Earth-Sun: The oldest three-body problem, Review of Modern Physics, 70(2), 589-639 (1998)

[9] Zhu Y., Updating the Historical Perspective of the Interaction of Gravitational Field and Orbit in Sun-Planet- 
Moon System, International J. of Astronomy and Astrophysics, 11, 343 (2021)

[10] Weissman P. R., The Oort Cloud, ASP conference series, 107 (1996)

[11] Schr"oder K.-P., \& Smith R. C., Distant future of the Sun and Earth revisited, Mon. Not. R. Astron. Soc. 000, 1$10(2008)$

[12] Fontaine G., Brassard P., Charpinet S., Randall S.K. and Van Grootel V., An overview of white dwarf stars, EPJ Web of Conferences 43, 05001 (2013)

[13] Issaoun S., Johnson M. D., Blackburn L., Brinkerink C. D., et al, The Size, Shape, and Scattering of Sagittarius A* at 86 GHz: First VLBI with ALMA, Astrophysical Journal 871.1(2019)

[14] Sartore N., Ripamonti E., Treves A., Turolla R., Space and velocity distributions of neutron stars in the Milky Way, Advances in Space Research, 47, 8 (2011)

[15] Dillon G., On the gravitational self-energy of a spherical shell, European Physical Journal Plus, 128(7):1-6(2013)

[16] Whitehead A., The Physics of Neutron Stars, Physics 518, (2009)

[17] Arsiwalla X., Boer J. D., Papadodimas K., \& Verlinde, E., Degenerate stars and gravitational collapse in ads/cft. Journal of High Energy Physics, 2011(1), 1-66(2010)

[18] Lai D., Ho W. Polarized X-Ray Emission from Magnetized Neutron Stars: Signature of Strong-Field Vacuum Polarization, Physical Review Letters, 91(7):071101 (2003)

[19] Mignani R. P., Testa V., Gonzalez Caniulef D., Taverna R., et al, Evidence for vacuum birefringence from the first optical-polarimetry measurement of the isolated neutron star RX J1856.5-3754, MNRAS, 465, 492-500 (2017)

[20] Ge M. Y., Ji L., Zhang S. N., Santangelo, A., \& Zhu Y. et al, Insight-hxmt firm detection of the highest energy fundamental cyclotron resonance scattering feature in the spectrum of gro j1008-57. The Astrophysical Journal, 899(1), L19 (2020)

[21] Reid, M.J., Menten, K.M., Zheng, X.W., Brunthaler, A., Moscadelli, L., Xu, Y., et al, Trigonometric Parallaxes of Massive Star-Forming Regions. VI. Galactic Structure, Fundamental Parameters, and Noncircular Motions, The Astrophysical Journal, 700, 137-148 (2009) 
Supplementary Information 1

The average energy density of a gravitational field

From Figure 1 and the energy density of $\mathrm{U}=\frac{g^{2}}{8 \pi G}$ and $g=\mathrm{G} \frac{M}{r^{2}}$, the average energy density of the field can be obtained from $\bar{U}=\frac{\int_{r}^{R} \frac{G M^{2}}{8 \pi r^{4}}}{R-r}$. As shown in Figure 1, $\mathrm{r}$ and $\mathrm{R}$ are the radius of the star and the field respectively. Therefore, there are three kinds of the average energy density of the field:

First, as discussed in the Figures 1 and 2 , as $\mathrm{dr} \rightarrow 0, \mathrm{R}=\mathrm{r}+\mathrm{dr} \approx \mathrm{r}, g=g(r) \approx g(r+d r)$, in the volume $\mathrm{dV}=\frac{4}{3} \pi\left[(r+d r)^{3}-r^{3}\right]$, the gravitational field can be treated as a uniform field. The average energy density can be approximately expressed as

$$
\bar{U}=\frac{g^{2}}{8 \pi G}
$$

Second, as $R-r$ need be considered, from $\bar{U}=\frac{\int_{r}^{R G M^{2}}}{R-r}$, there is

$$
\bar{U}=-\frac{1}{3} \frac{G M^{2}}{8 \pi R^{3}}-\frac{G M^{2}}{8 \pi r^{3}}
$$

From Figure 1 we know, Equation (s2) is the average energy density of the field in the shell of $\mathrm{V}(\mathrm{R})=\frac{4}{3} \pi\left(R^{3}-\right.$ $\left.r^{3}\right)$

Third, as $\mathrm{R}$ is very large relative to $\mathrm{r}, R-r \approx R$ and $\frac{G M^{2}}{8 \pi R^{3}}$ is very little relative to $\frac{G M^{2}}{8 \pi r^{3}}$. Thus, there is $\frac{G M^{2}}{8 \pi R^{3}}-$ $\frac{G M^{2}}{8 \pi r^{3}} \approx-\frac{G M^{2}}{8 \pi r^{3}}$. Therefore, (s2) approximately is $\bar{U}=\frac{1}{3} \frac{G M^{2}}{8 \pi r^{3} R}$. Let $g^{2}=G^{2} \frac{M^{2}}{r^{4}}$, it can be rewritten as

$$
\bar{U}=\frac{g^{2}}{24 \pi G} \frac{r}{R}
$$

